\title{
Biologia e exigências térmicas de Trichogramma pretiosum Riley (Hymenoptera, Trichogrammatidae) "linhagem bonagota" criado em ovos de Bonagota salubricola (Meyrick) (Lepidoptera, Tortricidae)
}

\author{
Patrik Luiz Pastori ${ }^{1}$, Lino Bittencourt Monteiro ${ }^{2} \&$ Marcos Botton $^{3}$
}

\begin{abstract}
${ }^{1}$ Departamento de Zoologia, Universidade Federal do Paraná, 81.531-990 Curitiba-PR, Brasil. plpastori@yahoo.com.br ${ }^{2}$ Departamento de Fitotecnia e Fitossanitarismo, Universidade Federal do Paraná, 80.035-050 Curitiba-PR, Brasil. lbmonteiro@terra.com.br ${ }^{3}$ Centro Nacional de Pesquisa Uva e Vinho, Empresa Brasileira de Pesquisa Agropecuária, 95.700-000 Bento Gonçalves-RS, Brasil. marcos@cnpuv.embrapa.br
\end{abstract}

\begin{abstract}
Biology and thermal requirements of Trichogramma pretiosum Riley (Hymenoptera, Trichogrammatidae) "strain bonagota" on eggs of Bonagota salubricola (Meyrick) (Lepidoptera, Tortricidae). The biology of Trichogramma pretiosum Riley (Hymenoptera, Trichogrammatidae) "strain bonagota" reared in eggs of Bonagota salubricola (Meyrick) (Lepidoptera, Tortricidae) was studied in laboratory estimating the thermal requirements and the number of annual generations in some apple production regions of the Rio Grande do Sul and Santa Catarina States. Eggs of B. salubricola were submitted to parasitism by $T$. pretiosum for $24 \mathrm{~h}$, and transferred to incubators regulated to $18,20,22,25,28,30$ and $32^{\circ} \mathrm{C}$. The duration of the biological cycle (egg-adult) was influenced by temperature ranging from 8.18 to 18.12 days at 32 and $18^{\circ} \mathrm{C}$, respectively. The parasitoid emergency was higher $(66.2 \%)$ at $22^{\circ} \mathrm{C}$. The sex ratio of $T$. pretiosum ranged from 0.61 to 0.72 being not influenced by temperature. The highest relation of parasitoids/egg was found at $30^{\circ} \mathrm{C}(1.41)$. The thermal constant $(\mathrm{k})$ and the threshold temperature $(\mathrm{Tb})$ was 187.7 degrees days and $7.42^{\circ} \mathrm{C}$, respectively. The number of annual generations estimated for T. pretiosum for Fraiburgo and São Joaquim, SC and Vacaria, RS was 17.5; 11.6 and 16.8 , respectively.
\end{abstract}

KEYWORDS. Biological control; Brazilian apple leafroller; egg parasitoids.

RESUMO. Biologia e exigências térmicas de Trichogramma pretiosum Riley (Hymenoptera, Trichogrammatidae) "linhagem bonagota" criado em ovos de Bonagota salubricola (Meyrick) (Lepidoptera, Tortricidae). Neste trabalho foi estudada a biologia de Trichogramma pretiosum Riley (Hymenoptera, Trichogrammatidae) "linhagem bonagota" criado em ovos de Bonagota salubricola (Meyrick) (Lepidoptera, Tortricidae), determinando-se as exigências térmicas e o número de gerações do parasitóide para diferentes regiões produtoras de maçã do Rio Grande do Sul e Santa Catarina. Ovos de B. salubricola com 24 horas de idade foram submetidos ao parasitismo de T. pretiosum durante 24 horas, sendo posteriormente transferidos para câmaras climatizadas reguladas nas temperaturas de $18,20,22,25,28,30$ e $32^{\circ} \mathrm{C}$. A duração do ciclo biológico (ovo-adulto) foi significativamente influenciada pela temperatura variando de 8,18 a 18,12 dias a 32 e $18^{\circ} \mathrm{C}$, respectivamente. A porcentagem de emergência dos parasitóides foi maior $(66,2 \%)$ a $22^{\circ} \mathrm{C}$. A razão sexual de $T$. pretiosum variou de 0,61 a 0,72 não sendo influenciada pela temperatura. A maior relação de parasitóides/ovo foi encontrada a $30^{\circ} \mathrm{C}(1,41)$. A constante térmica $(\mathrm{K})$ e a temperatura base $(\mathrm{Tb})$ foram de 187,7 graus dias e $7,42^{\circ} \mathrm{C}$, respectivamente. O número estimado de gerações anuais de T. pretiosum para os municípios de Fraiburgo e São Joaquim, SC e Vacaria, RS foi de 17,$5 ; 11,6$ e 16,8, respectivamente.

PALAVRAS-CHAVE. Controle biológico; Lagarta-enroladeira-da-maçã; parasitóide de ovos.

A lagarta-enroladeira-da-maçã Bonagota salubricola (Meyrick, 1937) (Lepidoptera, Tortricidae) anteriormente conhecida como Bonagota cranaodes e redescrita por Brown \& Razowski (2003) é considerada uma das principais pragas da macieira no Rio Grande do Sul e Santa Catarina, com perdas anuais entre 3 e $5 \%$. Considerando-se uma produção média de $40.000 \mathrm{~kg} / \mathrm{ha}$, o dano pode representar de 1200 a $2000 \mathrm{~kg} / \mathrm{ha} \mathrm{a}$ menos de frutos colhidos para comercialização in natura (Kovaleski et al. 1998).

A baixa eficiência do controle químico atualmente empregado (Kovaleski \& Ribeiro 2003), o desequilíbrio biológico causado pela aplicação de inseticidas de amplo espectro e as exigências cada vez maiores do mercado por produtos com ausência de resíduos tóxicos, torna fundamental a busca por métodos alternativos para o controle do inseto (Borba 2003).

Uma das opções para o controle dessa praga na cultura seria a utilização de inimigos naturais e, dentre eles, destacase o uso de parasitóides de ovos do gênero Trichogramma Westwood, 1833 (Hymenoptera, Trichogrammatidae) (Monteiro et al. 2004). Esses insetos parasitam ovos de inúmeras pragas agrícolas e florestais, principalmente da ordem Lepidoptera (Smith 1996; Hassan 1997), sendo atualmente utilizados no controle biológico de pragas-chave de 34 culturas em mais de 30 países, onde é liberado de forma inundativa, em cerca de 32 milhões de hectares (Hassan 1997; Pratissoli et al. 2003; Pratissoli et al. 2005ab; Molina \& Parra 2006). Os motivos do sucesso desses parasitóides no controle biológico são: a característica intrínseca deste inimigo natural ser um parasitóide de ovos, portanto controla a praga na sua fase inicial de desenvolvimento e a facilidade de criação em laboratório, o que permite a realização de estudos básicos da relação parasitóide-hospedeiro e estimar o seu potencial em campo (Parra et al. 1989). 
No Brasil poucos estudos visando o controle biológico de B. salubricola utilizando Trichogramma spp. foram realizados, uma vez que em levantamentos desses parasitóides de ovos em pomares comerciais não foi verificada a ocorrência dos mesmos, possivelmente devido sua sensibilidade aos inseticidas empregados (Fonseca 2001; Botton et al. 2002). Entretanto, mais recentemente, Monteiro et al. (2004) registraram a ocorrência de uma linhagem de Trichogramma pretiosum Riley, 1879 (Hymenoptera, Trichogrammatidae) parasitando naturalmente ovos de B. salubricola em pomares de macieira na região de Fraiburgo, SC, abrindo novas perspectivas para utilização deste inimigo natural na cultura.

O sucesso na introdução ou no desenvolvimento de programas de controle biológico com Trichogramma, depende do pleno conhecimento do potencial da espécie e/ou linhagem a ser empregada e do hospedeiro a ser controlado, além das exigências térmicas e da influência das condições climáticas predominantes (Parra 1997; Pratissoli \& Parra 2000, 2001; Pratissoli et al. 2003).

Neste trabalho foi estudado o desenvolvimento e determinadas as exigências térmicas de T. pretiosum, "linhagem bonagota", criado em ovos de B. salubricola estimando-se o número de gerações do parasitóide para localidades produtoras de maçãs do Rio Grande do Sul e Santa Catarina, visando fornecer subsídios para a utilização desta linhagem em programas de controle biológico na cultura da macieira.

\section{MATERIALE MÉTODOS}

O experimento foi conduzido no Laboratório de Manejo Integrado de Pragas (LMIP) do Setor de Ciências Agrárias da Universidade Federal do Paraná (SCA-UFPR) e constou das seguintes etapas:

Criação de B. salubricola. As posturas foram coletadas em pomares comerciais de macieira em Fraiburgo, $\mathrm{SC}\left(27^{\circ} 02^{\prime} \mathrm{S}\right.$; $\left.50^{\circ} 55^{\prime} \mathrm{W}\right)$ e acondicionadas em tubos de vidro $(8,5 \times 2,5 \mathrm{~cm})$, fechados com filme plástico de $\mathrm{PVC}^{\circledR}$, permanecendo em câmara climatizada à temperatura de $25 \pm 1^{\circ} \mathrm{C}, 70 \pm 10 \%$ de umidade relativa e fotofase de $14 \mathrm{~h}$ até a eclosão das lagartas. As lagartas eclodidas foram transferidas para tubos de vidro $(8,5 \times 2,5 \mathrm{~cm})$ e criadas segundo a metodologia de Parra et al. (1995), permitindo o ciclo completo de desenvolvimento dessa espécie em laboratório.

Coleta, manutenção e multiplicação de Trichogramma pretiosum "linhagem bonagota". Os exemplares de $T$. pretiosum "linhagem bonagota" utilizados eram provenientes da criação estoque do LMIP, coletados em pomares comerciais de macieiras localizados em Fraiburgo, SC. Para a coleta dos parasitóides foram recolhidas posturas de B. salubricola e transferidas para câmaras climatizadas à temperatura de $25 \pm 1^{\circ} \mathrm{C}$, $70 \pm 10 \%$ de umidade relativa e fotofase de 14 horas, a fim de observar os ovos parasitados e a emergência de adultos. Os parasitóides emergidos foram enviados ao Departamento de Entomologia, Fitopatologia e Zoologia Agrícola da Escola Superior de Agricultura “Luiz de Queiroz" Piracicaba, SP, para identificação pelo Dr. Roberto Antônio Zucchi.

Para a manutenção dos parasitóides, ovos de Sitotroga cerealella (Olivier, 1819) (Lepidoptera, Gelechiidae) obtidos segundo técnica adaptada de Hassan (1997) e Navarro (1998) foram colados com goma arábica diluída a $25 \%$ sobre retângulos de cartolina azul celeste $(8,0 \times 2,0 \mathrm{~cm})$. Estas cartelas foram inseridas em tubos de vidro $(8,5 \times 2,5 \mathrm{~cm})$, contendo adultos recém emergidos e, em seguida os tubos foram vedados com filme plástico de $\mathrm{PVC}^{\circledR}$, a fim de evitar a fuga dos parasitóides. O parasitismo foi permitido por 24 horas em sala climatizada nas mesmas condições descritas.

Desenvolvimento de $T$. pretiosum "linhagem bonagota" em diferentes temperaturas. Posturas contendo 30-40 ovos de B. salubricola com até 24 horas de desenvolvimento embrionário foram coletadas nas gaiolas de criação, transferidas para cápsulas de gelatina $(2,5 \times 0,5 \mathrm{~cm})$ e fechadas. Em seguida, foram introduzidas fêmeas de $T$. pretiosum recém-emergidas na proporção de 1 fêmea do parasitóide para 10 ovos da praga. O parasitismo foi permitido por 24 horas em câmara climatizada com as mesmas condições descritas acima. Ao final desse período, as fêmeas foram retiradas com auxilio de um pincel fino sob microscópio estereoscópico e os tubos transferidos para câmaras climatizadas reguladas para as temperaturas de $18,20,22,25,28,30$ e $32^{\circ} \mathrm{C}, 70 \pm 10 \%$ de umidade relativa (UR) e fotofase de 14 horas.

Nas temperaturas estudadas foram observados os seguintes parâmetros biológicos: duração do ciclo (ovoadulto), realizada por meio de observações diárias, sempre no mesmo horário a partir das 18:00 h; porcentagem de emergência, efetuada a partir da contagem dos ovos do hospedeiro que apresentavam orifício de saída dos adultos, vistos sob microscópio estereoscópico (10 x); razão sexual, calculada a partir da fórmula: $r s=\left(n^{\circ}\right.$. de fêmeas $/ n^{\circ}$. de fêmeas $+n^{\circ}$. de machos), sendo o sexo dos indivíduos determinado com base nas características morfológicas das antenas (Bowen \& Stern 1966) e o número de indivíduos emergidos por ovo.

O delineamento experimental foi inteiramente casualizado com sete temperaturas e 50 repetições por tratamento, constituídas por 50 cartelas com 30-40 ovos cada. Os dados foram submetidos à análise de variância e as médias comparadas pelo teste de Duncan ( $\mathrm{p} \leq 0,05)$, utilizando-se o programa computacional StatSoft, Inc. (2001), STATISTICA (data analysis software system), versão 6.

Determinação das exigências térmicas e estimativa do número de gerações de $T$. pretiosum linhagem bonagota. $O$ cálculo da temperatura base $(\mathrm{Tb})$ e constante térmica $(\mathrm{K})$, foram obtidos pelo método da hipérbole (Haddad et al. 1999), a partir do programa MOBAE (Modelos Bioestatísticos Aplicados a Entomologia), baseando-se na duração do ciclo (ovo-adulto) nas temperaturas testadas.

O número provável de gerações anuais de T. pretiosum foi estimado para os municípios de Fraiburgo (SC), São Joaquim (SC) e Vacaria (RS), baseando-se na temperatura média mensal destas localidades com 11, 50 e 20 anos, respectivamente, por meio da equação: $N G=\left\{T\left(T_{m}-T b\right) / K\right\}$, onde: $K=$ constante térmica, $\mathrm{T}_{\mathrm{m}}=$ temperatura média mensal para cada localidade estudada, $\mathrm{Tb}=$ temperatura base $\left({ }^{\circ} \mathrm{C}\right)$ e $\mathrm{T}=$ tempo (dias). Os dados de temperatura média mensal dos municípios foram obtidos na Estação Experimental de São Joaquim da Empresa de Pesquisa Agropecuária e Extensão Rural de Santa Catarina S.A. (São Joaquim, SC), AGF Anti-Granizo Fraiburgo LTDA. (Fraiburgo, SC) e Estação Experimental de Fruticultura Temperada da Embrapa Uva e Vinho (Vacaria, RS). 


\section{RESULTADOSEDISCUSSÃO}

A duração média do ciclo biológico (ovo-adulto) de $T$. pretiosum "linhagem bonagota" em ovos de $B$. salubricola apresentou relação inversa com a elevação da temperatura, verificando-se diferenças significativas entre as temperaturas de 18 a $30^{\circ} \mathrm{C}$ (Tabela I). O tempo médio de desenvolvimento dos parasitóides a $18^{\circ} \mathrm{C}$ foi 2,2 vezes maior que a $32^{\circ} \mathrm{C}$ (Tabela I). A influência da temperatura sobre a velocidade de desenvolvimento deste parasitóide foi relatada por diversos autores (Stern \& Atallah 1965; Russo \& Voegelé 1982; Yu et al. 1984; Calvin et al. 1984; Pratissoli \& Parra 2000; Fonseca et al. 2005; Borba et al. 2006; Pratissoli et al. 2006) e corroborada nesta pesquisa.

Os resultados de ciclo biológico de T. pretiosum obtidos diferem daqueles encontrados por Fonseca et al. (2005), que observaram ciclos de desenvolvimento de $T$. pretiosum linhagem $\mathrm{L}_{\mathrm{M}}$ em ovos de $B$. salubricola (=cranaodes) mais longos, em todas as temperaturas, sendo a maior diferença a $18^{\circ} \mathrm{C}$. A duração do ciclo biológico $(9,42$ dias), obtido neste estudo a $25^{\circ} \mathrm{C}$ (Tabela I), difere dos resultados de Borba et al. (2006), que trabalhando com duas linhagens de T. pretiosum $\left(\mathrm{L}_{3}\right.$ e $\left.\mathrm{L}_{4}\right)$ e uma de Trichogramma bruni Nagaraja, 1983 (Hymenoptera, Trichogrammatidae) $\left(\mathrm{L}_{9}\right)$ em ovos de $B$. salubricola, encontraram menor duração do período de desenvolvimento, 8,71; 8,50 e 8,50 dias, respectivamente, para as três linhagens/espécies. A variação na duração do ciclo (ovo-adulto) de espécies de Trichogramma em determinada faixa de temperatura registrado por diferentes autores indica, segundo Pratissoli et al. (2003), que este parâmetro biológico não só depende da temperatura utilizada, mas também da origem, do hospedeiro e da cultura em que foi coletado e da adaptação da espécie ou linhagem. Cabe ressaltar que as linhagens de T. pretiosum utilizadas por Fonseca et al. (2005) e por Borba et al. (2006) foram coletados em tomateiros e macieiras, respectivamente.

Os parasitóides deste estudo foram criados em ovos de $B$. salubricola, mas a duração do ciclo biológico (ovo-adulto) na faixa térmica 18 a $22^{\circ} \mathrm{C}$, quando comparada com resultados de outras pesquisas com diferentes espécies e/ou linhagens do parasitóide e hospedeiros (Bleicher \& Parra 1989; Pratissoli \& Parra 2000; Pereira et al. 2004; Zago et al. 2006) mostrou-se sempre inferior, com possível adaptação dessa linhagem ao hospedeiro e capacidade em aumentar sua população mesmo em temperaturas mais baixas, características das regiões produtoras do sul do Brasil. No entanto, na faixa térmica 25 a $32^{\circ} \mathrm{C}$, os resultados obtidos neste estudo foram semelhantes aos observados por Bleicher \& Parra (1989), Pratissoli \& Parra (2000), Pereira et al. (2004) e Zago et al. (2006).

A porcentagem de emergência de T. pretiosum em ovos de B. salubricola foi influenciada pela variação da temperatura, sendo que na faixa térmica 20 a $25^{\circ} \mathrm{C}$ observaram-se taxas de viabilidade superiores a $60 \%$ (Tabela I). A menor viabilidade foi observada a $18^{\circ} \mathrm{C}(53,5 \%)$, entretanto não diferiu significativamente das demais temperaturas (Tabela I). As taxas de emergência de $T$. pretiosum linhagem bonagota, inferiores a $60 \%$ na temperatura de $18^{\circ} \mathrm{C}$ e acima de $28^{\circ} \mathrm{C}$ denotam que a faixa entre $20^{\circ} \mathrm{C}$ e $25^{\circ} \mathrm{C}$ é a mais adequada à criação do parasitóide em laboratório. Resultados semelhantes foram obtidos por Fonseca et al. (2005) utilizando T. pretiosum linhagem $\mathrm{L}_{\mathrm{M}}$ em ovos de $B$. salubricola, que observaram efeito prejudicial de temperaturas próximas a $18^{\circ} \mathrm{C}$. Os resultados obtidos neste estudo ainda corroboram com os de Cônsoli \& Parra (1995), que conduzindo estudos com Trichogramma galloi Zucchi, 1988 (Hymenoptera, Trichogrammatidae) em ovos de Diatraea saccharalis (Fabricius, 1794) (Lepidoptera, Crambidae) observaram que a emergência foi drasticamente reduzida em temperaturas abaixo de $18^{\circ} \mathrm{C}$. A percentagem de emergência observada a $25^{\circ} \mathrm{C}(61,06 \%)$ é semelhante ao relatado por Borba et al. (2006).

A razão sexual de $T$. pretiosum variou de 0,72 a 0,61 para as temperaturas de 32 e $22^{\circ} \mathrm{C}$, respectivamente (Tabela I). Estes resultados diferem dos obtidos por Fonseca et al. (2005), que observaram maior número de fêmeas a $14 \mathrm{e} 28^{\circ} \mathrm{C}$ e de Pereira $e t$ al. (2004) que utilizando Trichogramma exiguum Pinto \& Platner, 1978 (Hymenoptera, Trichogrammatidae) em ovos de Plutella xylostella (Linnaeus, 1758) (Lepidoptera, Plutellidae) observaram maior número de fêmeas a $22^{\circ} \mathrm{C}$. Já Zago et al. (2006) utilizando Trichogramma pratissolii Querino \& Zucchi, 2003 (Hymenoptera, Trichogrammatidae) em ovos de Anagasta kuehniella (Zeller, 1879) e Corcyra cephalonica (Stainton, 1865) (Lepidoptera, Pyralidae) observaram maior número de fêmeas em temperaturas mais elevadas. Entretanto, não foram observados efeitos da temperatura na razão sexual de linhagens de T. pretiosum em ovos de Tuta absoluta (Meyrick, 1917) e Phthorimaea operculella (Zeller, 1873) (Lepidoptera,

Tabela I. Ciclo biológico (Dias \pm EP) (ovo-adulto), emergência $(\% \pm$ EP), razão sexual (Médias \pm EP) e número médio (Médias \pm EP) de Trichogramma pretiosum Riley (Hymenoptera, Trichogrammatidae) "linhagem bonagota" emergidos por ovo de Bonagota salubricola (Meyrick) (Lepidoptera, Tortricidae) em diferentes temperaturas. UR: $70 \pm 10 \%$, fotofase $14 \mathrm{~h}$.

\begin{tabular}{ccccc}
\hline $\begin{array}{c}\text { Temperatura } \\
\left({ }^{\circ} \mathrm{C}\right)\end{array}$ & $\begin{array}{c}\text { Duração } \\
(\text { Dias } \pm \mathrm{EP})\end{array}$ & $\begin{array}{c}\text { Emergência } \\
(\% \pm \mathrm{EP})\end{array}$ & $\begin{array}{c}\text { Razão sexual } \\
(\text { Médias } \pm \mathrm{EP})\end{array}$ & $\begin{array}{c}\mathrm{N}^{\circ} \text { de parasitóides/ovo } \\
(\text { Médias } \pm \mathrm{EP})\end{array}$ \\
\hline 18 & $18,12 \pm 0,22 \mathrm{a}^{1}(16,0-22,0)^{2}$ & $53,52 \pm 2,84 \mathrm{~b}^{1}$ & $0,66 \pm 0,03 \mathrm{abc}^{1}$ & $1,15 \pm 0,03 \mathrm{~b}^{1}$ \\
20 & $17,40 \pm 0,13 \mathrm{~b}(16,0-20,0)$ & $61,67 \pm 2,76 \mathrm{ab}$ & $0,69 \pm 0,02 \mathrm{ab}$ & $1,21 \pm 0,02 \mathrm{~b}$ \\
22 & $12,38 \pm 0,15 \mathrm{c}(12,0-18,0)$ & $66,16 \pm 2,50 \mathrm{a}$ & $0,61 \pm 0,02 \mathrm{c}$ & $1,39 \pm 0,06 \mathrm{a}$ \\
25 & $9,42 \pm 0,10 \mathrm{~d}(8,0-11,0)$ & $61,06 \pm 3,34 \mathrm{ab}$ & $0,64 \pm 0,02 \mathrm{abc}$ & $1,09 \pm 0,02 \mathrm{~b}$ \\
28 & $8,96 \pm 0,09 \mathrm{e}(8,0-11,0)$ & $57,11 \pm 2,49 \mathrm{~b}$ & $0,62 \pm 0,03 \mathrm{bc}$ & $1,16 \pm 0,02 \mathrm{~b}$ \\
30 & $8,20 \pm 0,09 \mathrm{f}(7,0-10,0)$ & $56,03 \pm 2,61 \mathrm{~b}$ & $0,69 \pm 0,02 \mathrm{ab}$ & $1,41 \pm 0,15 \mathrm{a}$ \\
32 & $8,18 \pm 0,08 \mathrm{f}(7,0-9,0)$ & $55,91 \pm 2,62 \mathrm{~b}$ & $0,72 \pm 0,02 \mathrm{a}$ & $1,13 \pm 0,02 \mathrm{~b}$ \\
\hline
\end{tabular}

${ }^{1}$ Médias seguidas de mesma letra, na coluna, não diferem entre si, pelo Teste de Duncan, ao nível de $5 \%$ de probabilidade.

${ }^{2}$ Números entre parênteses indicam o intervalo de variação. 


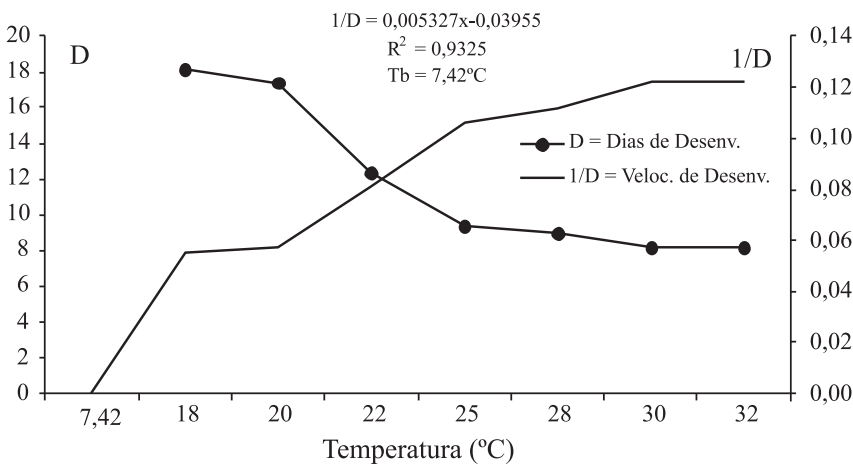

Fig. 1. Velocidade de desenvolvimento de Trichogramma pretiosum Riley (Hymenoptera, Trichogrammatidae) "linhagem bonagota" em ovos de Bonagota salubricola (Meyrick) (Lepidoptera, Tortricidae), em diferentes temperaturas. UR: $70 \pm 10 \%$, fotofase $14 \mathrm{~h}$.

Gelechiidae) (Pratissoli \& Parra 2000) e em ovos de $A$. kuehniella (Bleicher \& Parra 1989). Segundo Bowen \& Stern (1966) a temperatura interfere na razão sexual de Trichogramma, podendo-se obter maior proporção de machos em temperaturas superiores a $30^{\circ} \mathrm{C}$, fato não observado nesta pesquisa.

O número médio de parasitóides emergidos por ovo de $B$. salubricola foi sempre superior a um em todas as temperaturas estudadas (Tabela I). Os maiores valores, 1,41 e 1,39, foram observados a 30 e $22^{\circ} \mathrm{C}$, respectivamente, diferindo significativamente. Na temperatura $25^{\circ} \mathrm{C}$ foi observada emergência de 1,09 parasitóides por ovo de B. salubricola, valor próximo ao encontrado por Fonseca (2001) para quatro linhagens de T. pretiosum e por Borba et al. (2006) para duas linhagens de T. pretiosum e uma de T. bruni, sendo que ambos os autores utilizaram B. salubricola. Fonseca et al. (2005) verificaram que o maior número de T. pretiosum linhagem $\mathrm{L}_{\mathrm{M}}$ por ovo de B. salubricola foi obtido a $28^{\circ} \mathrm{C}$. Já Pratissoli \& Parra (2000), com T. pretiosum em ovos de P. operculella, observaram maiores valores a $25^{\circ} \mathrm{C}$. De acordo com Alencar $e t$ al. (2000), o número de Trichogramma spp. que se desenvolve por ovo do hospedeiro é variável, sendo proporcional ao tamanho do mesmo. Esse fato foi constatado por Parra \& Zucchi (1986) e Silva \& Hohmann (1988), que observaram, respectivamente, a emergência de um indivíduo por ovo de $S$. cerealella e 45 indivíduos por ovo de Erinnys ello (Linnaeus, 1758) (Lepidoptera, Sphingidae).

Com base no desenvolvimento do ciclo biológico (ovoadulto) de $T$. pretiosum nas temperaturas de 18, 20, 22, 25, 28, 30 e $32^{\circ} \mathrm{C}$ foi determinado o limite térmico inferior de desenvolvimento $(\mathrm{Tb})$ e a constante térmica $(\mathrm{K})$ em ovos de $B$. salubricola de $7,42^{\circ} \mathrm{C}$ e 187,7 graus-dias, respectivamente (Fig. 1). Estes resultados foram próximos aos obtidos por Zago et al. (2006) para T. pratissolii em ovos de A. kuehniella e $C$. cephalonica, os de Pereira et al. (2004) para T. exiguum e T. pretiosum em ovos de P. xylostella e os de Pratissoli et al. (2006) para cinco linhagens de T. pretiosum em ovos de $T$. absoluta, com variações ligeiramente inferiores. Fonseca $e t$ al. (2005) obtiveram uma temperatura base de $11,9^{\circ} \mathrm{C}$, com uma constante térmica de 153,4 graus-dias para $T$. pretiosum linhagem $\mathrm{L}_{\mathrm{M}}$ quando avaliaram o desenvolvimento deste parasitóide em ovos de B. salubricola. Ressalta-se que as variações entre os valores da temperatura base $(\mathrm{Tb})$ e

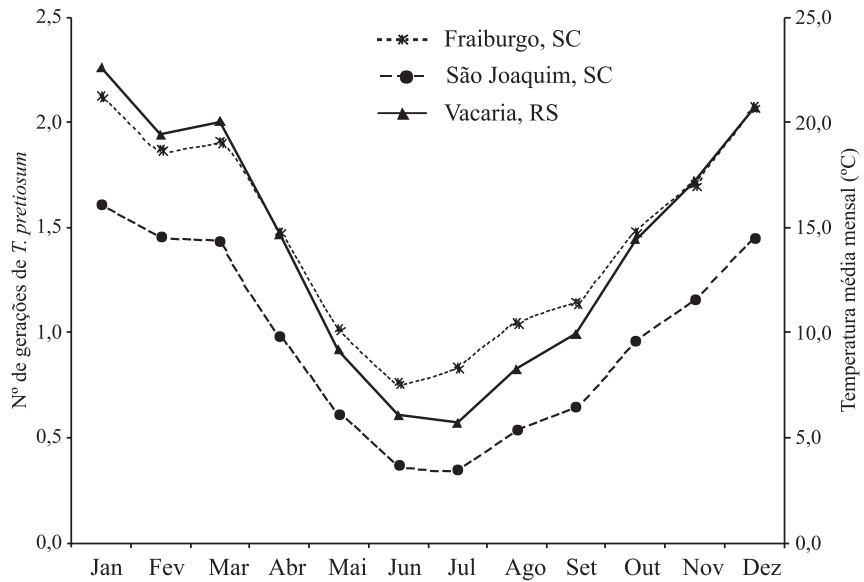

Fig. 2. Estimativa do número de gerações/mês de Trichogramma pretiosum Riley (Hymenoptera, Trichogrammatidae) "linhagem bonagota" criados em ovos de Bonagota salubricola (Meyrick) (Lepidoptera, Tortricidae), para os municípios de Fraiburgo e São Joaquim (SC) e Vacaria (RS).

constante térmica $(\mathrm{K})$ indicam a influência da espécie, linhagem e hospedeiro de criação (Russo \& Voegelé 1982; Goodenough et al. 1983; Calvin et al. 1984; Bleicher \& Parra 1990; Pereira et al. 2004) demonstrando que T. pretiosum "linhagem bonagota" seria mais adaptada a baixas temperaturas provavelmente por já ser originaria da região produtora e da praga alvo.

$\mathrm{O}$ número estimado de gerações anuais de T. pretiosum para o município de Fraiburgo, SC foi de 17,5 ao longo do ano, enquanto para o município de São Joaquim, SC, foi de 11,6 e para o município de Vacaria, RS, estimaram-se aproximadamente 16,8 gerações para T. pretiosum linhagem bonagota (Fig. 2). Através da comparação das exigências térmicas de $B$. salubricola (temperatura base e constante térmica de $6,8^{\circ} \mathrm{C}$ e 745 graus dias, respectivamente) obtidos por Botton et al. (2000), com as exigências térmicas de T. pretiosum "linhagem bonagota" obtidos nesta pesquisa, pode-se prever que, ao longo do ano, o número de gerações desses parasitóides será sempre maior que o de B. salubricola, característica esta importante para um programa de controle biológico.

Agradecimentos. Ao Conselho Nacional de Desenvolvimento Científico e Tecnológico (CNPq) pela concessão de bolsas, a Universidade Federal do Paraná e Embrapa Uva e Vinho, por possibilitarem a execução desta pesquisa e a Estação Experimental de São Joaquim da EPAGRI, AGF Anti-Granizo Fraiburgo LTDA. e Estação Experimental de Fruticultura Temperada da Embrapa Uva e Vinho pelas informações metereológicas.

\section{REFERÊNCIAS}

Alencar, J. A.; F. N. P. Haji; J. V. Oliveira \& A. N. Moreira. 2000. Biologia de Trichogramma pretiosum Riley em ovos de Sitotroga cerealella (Olivier). Pesquisa Agropecuaria Brasileira 35: 1669-1674.

Bleicher, E. \& J. R. P. Parra. 1989. Espécies de Trichogramma parasitóides de Alabama argillacea. I. Biologia de três populações. Pesquisa Agropecuaria Brasileira 24: 929-940.

Bleicher, E. \& J. R. P. Parra. 1990. Espécies de Trichogramma parasitóides de Alabama argillacea. III. Determinação das exigências térmicas de três populações. Pesquisa Agropecuaria Brasileira 25: 215-219. 
Borba, R. S. 2003. Biologia de Trichogramma pretiosum e T. bruni (Hymenoptera: Trichogrammatidae) em ovos de Bonagota cranaodes (Lepidoptera: Tortricidae) e suas diferenciações através de marcadores moleculares. Pelotas, Dissertação de Mestrado, UFPel, 54 p. e 2.

Borba, R. S.; M. S. Garcia; A. Kovaleski; A. Comioto \& R. L. Cardoso. 2006. Biologia e exigências térmicas de Trichogramma pretiosum Riley (Hymenoptera: Trichogrammatidae) sobre ovos de Bonagota cranaodes (Meyrick) (Lepidoptera: Tortricidae). Ciência Rural 36: $1345-1352$.

Botton, M.; O. Nakano \& A. Kovaleski. 2000. Exigências térmicas e estimativa do número de gerações de Bonagota cranaodes (Meyrick) (Lepidoptera: Tortricidae) em regiões produtoras de maçã do sul do Brasil. Anais da Sociedade Entomológica do Brasil 29: 633-637.

Botton, M.; O. Nakano \& A. Kovaleski. 2002. Parasitóides associados à lagarta-enroladeira Bonagota cranaodes (Meyrick, 1937) (Lepidoptera: Tortricidae) na cultura da macieira. Ciência Rural 32: $341-343$

Bowen, W. R. \& V. M. Stern. 1966. Effect of temperature on the production of males and sexual mosaics in a uniparental race of Trichogramma semifumatum (Hymenoptera: Trichogrammatidae). Annals of the Entomological Society of America 59: 823834.

Brown, J. W. \& J. Razowski. 2003. Description of Ptychocroca, a new genus from Chile and Argentina, with comments on the Bonagota Razowski group of genera (Lepidoptera: Tortricidae: Euliini). Zootaxa 303: $1-31$.

Calvin, D. D.; M. C. Knapp; S. M. Welch; F. L. Poston \& R. J. Elzinga. 1984. Impact of environmental factors on Trichogramma pretiosum reared on southwestern corn borer eggs. Environmental Entomology 13: 774-780.

Cônsoli, F. L \& J. R. P. Parra. 1995. Effects of constant and alternating temperatures on Trichogramma galloi Zucchi (Hym.: Trichogrammatidae) biology II. Parasitism capacity and longevity. Journal of Applied Entomology 119: 667-670.

Fonseca, F. L. 2001. Ocorrência de Trichogramma (Hymenoptera: Trichogrammatidae) nas localidades de Pelotas, Bento Gonçalves e Vacaria-RS e aspectos bioecológicos em ovos de Bonagota cranaodes (Meyrick, 1937) (Lepidoptera: Tortricidae). Pelotas, Dissertação de Mestrado, UFPel, 59 p.

Fonseca, F. L.; A. Kovaleski; J. Foresti \& R. Ringenberg. 2005. Desenvolvimento e exigências térmicas de Trichogramma pretiosum Riley (Hymenoptera: Trichogrammatidae) em ovos de Bonagota cranaodes (Meyrick) (Lepidoptera: Tortricidae). Neotropical Entomology 34: 945-949.

Goodenough, J. L.; A. W. Harstack \& E. G. King. 1983. Developmental models of Trichogramma pretiosum (Hymenoptera: Trichogrammatidae) reared on for hosts. Journal of Economic Entomology 76: 1095-1102.

Haddad, M. L.; J. R. P. Parra \& R. C. B. Moraes. 1999. Métodos para estimar os limites térmicos inferior e superior de desenvolvimento de insetos. Piracicaba, FEALQ, 29 p.

Hassan, S. A. 1997. Criação da traça do milho, Sitotroga cerealella, para a produção massal de Trichogramma, p. 173-182. In: J. R. P. Parra \& R. A. Zucchi (eds.). Trichogramma e o controle biológico aplicado. Piracicaba, FEALQ, 324 p.

Kovaleski, A.; M. Botton; A. E. Eiras \& E. F. Vilela. 1998. Lagartaenroladeira da macieira: Bioecologia e controle. Bento Gonçalves, Embrapa Uva e Vinho, 22 p. (Embrapa CNPUV. Circular Técnica, 24).

Kovaleski, A. \& L. G. Ribeiro. 2003. Manejo de pragas na produção integrada de maçã, p. 61-68. In: Protas, J. F. S. da \& R. M. V. Sanhueza (eds.). Produção integrada de frutas: $O$ caso da maçã no Brasil. Bento Gonçalves, Embrapa Uva e Vinho, 192 p.

Molina, R. M. da S. \& J. R. P. Parra. 2006. Seleção de linhagens de Trichogramma (Hymenoptera, Trichogrammatidae) e determinação do número de parasitóides a ser liberado para o controle de Gymnandrosoma aurantianum Lima (Lepidoptera, Tortricidae). Revista Brasileira de Entomologia 50: 534-539.

Monteiro, L. B.; A. de Souza; E. L. Belli; R. B. Q. da Silva \& R. A. Zucchi. 2004. Ocorrência de Trichogramma pretiosum Riley (Hymenoptera: Trichogrammatidae) em ovos de Bonagota cranaodes (Meyrick) (Lepidoptera: Tortricidae) em macieira Revista Brasileira de Fruticultura 26: 171-172.

Navarro, M. A. 1998. Trichogramma spp.: Produción, uso y manejo en Colombia. Valle del Cauca, Guadalajara de Buga, $176 \mathrm{p}$.

Parra, J. R. P. 1997. Técnicas de criação de Anagasta kuehniella, hospedeiro alternativo para produção de Trichogramma, p. 121150. In: J. R. P. Parra \& R. A. Zucchi (eds.). Trichogramma e o controle aplicado. Piracicaba, FEALQ, $324 \mathrm{p}$.

Parra, J. R. P. \& R. A. Zucchi. 1986. Uso de Trichogramma no controle de pragas, p. 54-75. In: Nakano, O.; S. Silveira Neto; J. R. P. Parra \& R. A. Zucchi (eds.). Atualização sobre métodos de controle de pragas. Piracicaba, FEALQ, $129 \mathrm{p}$.

Parra, J. R. P.; J. R. S. Lopes; E. Biral \& P. C. Gouveia. 1989. Número ideal de ovos de Anagasta kuehniella (Zeller, 1879) por caixa de criação para pesquisas com Trichogramma spp. Anais da Sociedade Entomológica do Brasil 18: 391-402.

Parra, J. R. P.; A. E. Eiras; M. L. Haddad; E. F. Vilela \& A. Kovaleski. 1995. Técnica de criação de Phtheochroa cranaodes Meyrick (Lepidoptera: Tortricidae) em dieta artificial. Revista Brasileira de Biologia 55: 537-543.

Pereira, F. F.; R. Barros; D. Pratissoli \& J. R. P. Parra. 2004. Biologia e exigências térmicas de Trichogramma pretiosum Riley e $T$. exiguum Pinto \& Platner (Hymenoptera: Trichogrammatidae) criados em ovos de Plutella xylostella (L.) (Lepidoptera: Plutellidae) Neotropical Entomology 33: 231-236.

Pratissoli, D. \& J. R. P. Parra. 2000. Desenvolvimento e exigências térmicas de Trichogramma pretiosum Riley, criados em duas traças do tomateiro. Pesquisa Agropecuária Brasileira 35: 12811288 .

Pratissoli, D. \& J. R. P. Parra. 2001. Seleção de linhagens de Trichogramma pretiosum Riley (Hymenoptera: Trichogrammatidae) para o controle das traças Tuta absoluta (Meyrick) e Phthorimaea operculella (Zeller) (Lepidoptera: Gelechiidae). Neotropical Entomology 30: 277-282.

Pratissoli, D.; E. F. Reis; H. B. Zago; P. L. Pastori \& T. Tamanhoni. 2006. Biologia e exigências térmicas de cinco linhagens de Trichogramma pretiosum Riley (Hymenoptera: Trichogrammatidae) criadas em ovos de Tuta absoluta (Meyrick) (Lepidoptera: Gelechiidae). Ciência Rural 36: 1671-1677.

Pratissoli, D.; J. C. Zanuncio; U. R. Vianna; J. S. Andrade; T. B. M. Pinon \& G. S. Andrade. 2005a. Thermal requirements of Trichogramma pretiosum and T. acacioi (Hym.: Trichogrammatidae), parasitoids of the avocado defoliator Nipteria panacea (Lep.: Geometridae), in eggs of two alternative hosts Brazilian Archives of Biology and Technology 48: 523-529.

Pratissoli, D.; M. J. Fornazier; A. M. Holtz; J. R. Gonçalves; A. B Chioramital \& H. B. Zago. 2003. Ocorrência de Trichogramma pretiosum em áreas comerciais de tomate, no Espírito Santo, em regiões de diferentes altitudes. Horticultura Brasileira 21: 73 76.

Pratissoli, D.; U. R. Vianna; H. B. Zago \& P. L. Pastori. 2005b Capacidade de dispersão de Trichogramma em tomateiro estaqueado. Pesquisa Agropecuária Brasileira 40: 613-616.

Russo, J. \& J. Voegelé. 1982. Influence de la temperature sur quatre espèces de trichogrammes (Hym.: Trichogrammatidae) parasites de la pyrale du mais Ostrinia nubialis Hubn. (Lep.: Pyralidae). 1. Dévelopment préimaginal. Agronomie 2: 509-516.

Silva, S. M. T. \& C. L. Hohmann. 1988. Parasitismo de ovos de Erinny ello (L.) (Lepidoptera: Sphingidae) em mandioca por Trichogramma spp. Anais da Sociedade Entomológica do Brasil 17: 87-93.

Smith, S. M. 1996. Biological control with Trichogramma: Advances, successes, and potential of their use. Annual Review of Entomology 41: 375-406.

Stern, V. M. \& Y. H. Atallah. 1965. Ecological studies of Trichogramma medicaginis, and emergence of two Trichogramma species from the same egg. Annals of the Entomological Society of America 58: $76-81$

Zago, H. B.; D. Pratissoli; R. Barros \& M. G. C. Gondim Jr. 2006. Biologia e exigências térmicas de Trichogramma pratissolii Querino \& Zucchi (Hymenoptera: Trichogrammatidae) em hospedeiros alternativos. Neotropical Entomology 35: 377-381.

Yu, D. S. K.; E. A. C. Hagley \& J. E. Laing. 1984. Biology of Trichogramma minutum Riley collected from apples in Southern Ontario. Environmental Entomology 13: 1324-1329.

Recebido em 10/09/2007; aceito em 20/05/2008 